\title{
Personality Assessment Inventory's Utility in Pre-Treatment Assessment for Violent Offenders
}

\author{
Randall Nedegaard, Travis Cronin \\ Department of Social Work Education, California State University, Fresno, CA, USA \\ Email: nedegaard@csufresno.edu, travis@csufresno.edu
}

How to cite this paper: Nedegaard, R., \& Cronin, T. (2021). Personality Assessment Inventory's Utility in Pre-Treatment Assessment for Violent Offenders. Open Journal of Social Sciences, 9, 169-180. https://doi.org/10.4236/jss.2021.96014

Received: May 21, 2021

Accepted: June 19, 2021

Published: June 22, 2021

Copyright $\odot 2021$ by author(s) and Scientific Research Publishing Inc. This work is licensed under the Creative Commons Attribution International License (CC BY 4.0).

http://creativecommons.org/licenses/by/4.0/ (c) (i) Open Access

\begin{abstract}
Domestic Violence programs are often mandated to treat perpetrators of intimate partner violence (IPV), yet ways to improve the effectiveness of these programs are needed. One possibility is to provide a more comprehensive assessment and screening so that group facilitators can be better prepared to serve their clientele from the very beginning of treatment. To that end, the Personality Assessment Inventory (PAI) was administered to 154 IPV perpetrators as part of pre-treatment assessment for group treatment programs. After the treatment groups were finished, Interviews were conducted with group facilitators to determine if the facilitators of groups for men who engage in IPV perceived the PAI as an effective pre-treatment assessment tool. The majority of the program facilitators believed the PAI to be a useful tool and discussed various ways they were able to use the results in a positive manner. Those who did not find it useful were likely not to take the time to use the PAI at all, indicating there was not enough time because of the arduous nature of the job. The PAI protocols collected from program participants are also presented and compared to those currently published in the literature for this population. PAI profiles for this group differed from the comparison groups in two ways. Within the clinical scales, this group scored higher than the community norms and the published norms for men engaging in IPV on negative relations, antisocial behaviors and alcohol problems. On the validity scales, they had a significantly higher number of invalid profiles, mainly due to higher levels of positive impression management.
\end{abstract}

\section{Keywords}

Intimate Partner Violence, Psychological Assessment, Perpetrator Treatment, Personality Assessment Inventory 


\section{Introduction}

Over the last 30 - 40 years, legal systems have been reformed to criminalize domestic violence and mandate prosecution protocols and courts order offenders to attend treatment programs. Unfortunately, some of these programs suffer from questionable levels of effectiveness (e.g., Babcock, Greene, \& Robie, 2004; Cheng, Davis, Jonson-Reid, \& Yaeger, 2019) due to a variety of factors from treatment attrition, to lack of fit between client needs, and treatment program methods or objectives (Meade, 2006).

Several treatment methods have been developed over the years and have been adopted by domestic violence agencies who are tasked to provide services for perpetrators of intimate partner violence (IPV). For example, the Duluth Model developed by Pence and Paymar (1993) out of the Domestic Abuse Intervention Program has been one of the most commonly used interventions in the United States for men who are court-ordered to treatment after conviction for IPV (Corvo, Dutton, \& Chen, 2009). This model is most famous for the development of the Power and Control Wheel that illustrates how perpetrators establish power and control over their partners through a range of behaviors. The Duluth model is a structured program that is largely psychoeducational while incorporating some cognitive-behavioral techniques (Bohall, Bautista, \& Musson, 2016).

Beyond the development of treatment programs that were primarily developed based on feminist theory and sociocultural concepts of domination and control, other tools have been used to better understand the motivations for violent behavior and the risk of recidivism of IPV perpetrators. For example, comprehensive personality inventories have been employed to research and develop relatively useful perpetrator typologies (e.g., Delsol, Margolin, \& John, 2003), inform forensic decision-making (e.g., Edens, Cruise, \& Buffington-Vollum, 2001), and predict offender misconduct (e.g., Magyar et al., 2012), and predict recidivism and future violence (e.g., Gardner, Boccaccini, Bitting, \& Edens, 2015). However, one underrepresented research area is the use of comprehensive personality assessments such as the Personality Assessment Inventory (PAI; Morey, 1991) to help augment programs for IPV perpetrators. In particular, using these tools for initial screening could better inform treatment program facilitators about the perpetrators they serve. This approach may help clarify treatment goals, and customize treatment plans.

The present study was developed as a way to test the value of using comprehensive personality assessments as part of the routine assessment process for two domestic violence offender treatment programs who were using the Duluth model. The purpose of the study was to examine the value of the PAI as a pre-treatment assessment tool for domestic violence offender treatment. Specifically, the researchers hoped to answer the questions, do group facilitators perceive the PAI as an effective tool as part of the pre-treatment assessment protocol for their group intervention with men engaging in IPV, and how do the PAI protocols collected from these programs compare to those currently pub- 


\section{lished in the literature for this population?}

\section{Literature Review}

The PAI has scales specifically designed to better understand and predict aggression (aggression subscale) and violence potential (violence potential index). Additionally, scales designed to measure antisocial features are expected to help determine misconduct and violence. Gardner, Boccaccini, Bitting, \& Edens (2015), conducted a meta-analysis of more than 30 studies to examine the ability of the PAI to predict institutional infractions and criminal recidivism among individuals in both treatment and correctional settings. Overall, the findings provided evidence that some scales had moderate predictive validity for institutional misconduct and somewhat lower predictive validity for recidivism.

A small number of studies have been published where the PAI has been used to assess offenders in forensic and treatment capacities. Morey \& Quigley (2002) discuss the benefits of assessing offenders with the PAI. Of specific note, they highlight the value of the PAI in augmenting risk appraisal and treatment planning. They provide a case example to illustrate how particular results from the PAI can inform case formulation and treatment planning. Additionally, Chambers and Wilson (2007) published an article where they used the PAI to assess male batterers. They sought to replicate earlier findings where IPV perpetrators were categorized into three clusters; borderline/dysphoric, "normal" and non-elevated, and antisocial/narcissistic. Their findings supported the first two groups and partially supported the third. They also found a subgroup of men who engaged in positive impression management who were significantly underreporting their symptoms. This group made up nearly $8 \%$ of their sample.

Most recently, the literature involving the PAI with offenders has been focusing on using it to identify interpersonal characteristics and antecedents to treatment success with sex offenders (Pappas, 2021; Parker, Mulay, \& Gottfried, 2020), predictors of treatment success and classification of juvenile offenders (Charles, Floyd, Bulla, Barry, \& Anestis, 2021; Humenik, Sherrill, Kantor, \& Dolan, 2019), and to better understand and treat female offenders (Cunliffe, 2019; Miller \& Marshall, 2019).

\section{Methods}

\subsection{Participants}

As is common for many domestic violence offender treatment programs, the majority of the participants are court sanctioned to treatment following conviction of a domestic assault offense. In this study, $95 \%$ of the participants were court-ordered to the treatment programs. Fifty-five percent of the sample was white, $17 \%$ were African American, 13\% were Native American, 7\% were Hispanic, $4 \%$ were Asian, and $4 \%$ were 2 or more races. This means that forty-five percent of the sample were non-white which may not seem problematic at first glance, but the Census Bureau indicates the racial composition of the areas 
where these treatment programs were providing services was $86 \%$ white during the most recent American Community Survey (Census Bureau, 2021). This suggests that persons of color were highly overrepresented within this particular court system. Unfortunately, this is consistent with findings both within the US (e.g., Jeremiah \& Oyewuwo-Gassikia, 2019) and internationally (e.g., Douglas \& Fitzgerald, 2018) that demonstrate a disproportionate number of people of color are named on domestic violence orders, charged when they break them, and are significantly more likely than their white counterparts to receive a sentence of imprisonment for a contravention of an order for protection.

Thirty-five percent of the sample fell between the ages of $18-29,37 \%$ were between 30 - 39, 22\% were between $40-49,5 \%$ were between $50-59$ and $1 \%$ were 60 or over. Forty-seven percent of the participants lived in poverty as they indicated an annual income of less than $\$ 10,000$ per year. Over a third (35\%) indicated alcohol or drug involvement and $18 \%$ indicated they were on some sort of disability status.

\subsection{Procedures}

Two small Midwest domestic violence treatment programs opted to utilize the PAI as a part of their intake process over the course of two years to administer to the offenders who were participating in a group treatment program for IPV. The PAI was administered as a part of the normal intake assessment by the DV program administrator who was trained by the lead author. The score sheets were then delivered to the lead author who entered them into the PAI software portfolio for scoring. The lead author then provided an individualized clinical interpretive report based on data provided from the PAI software portfolio back to the program director for dissemination to the appropriate group facilitator. Over the course of the project, 4 group facilitators were interviewed about their perceptions regarding the value of adding the Personality Assessment Inventory to their intake process. While the Duluth Model is highly standardized and the facilitators were not allowed to customize their interventions, it was thought that having individualized assessment data would allow facilitators to emphasize aspects of the group treatment program that could have a potentially larger impact on the areas of concern that were identified by the PAI assessments. It was also thought that it could help the facilitators to identify individuals with significant positive impression management issues or with abnormally high treatment rejection scores. If the individual's behavior in group was consistent with their scores in these areas, it could help facilitators to make decisions about advancing the group members if they were making poor or minimal progress.

\subsection{Analysis Method}

Two methods were used for this study. With regard to the interview data from the group facilitators, content analysis systematically identifies and categorizes data into codes, or meaningful pieces of content, in order to develop significant 
themes in qualitative analysis (Hsieh \& Shannon, 2005; Patton, 2002). The researchers used content analysis for the interview data. For the study, the interview data was coded independently by two coders. The researchers each coded the interview data to identify preliminary words or phrases that described a group facilitator's experience. This process produced substantial intercoder reliability. The researchers then discussed, and agreed on, the main themes which are described later. The main themes were influenced by the structure of the interview, but this act was still an important part of the process.

Data from the PAI was collected from the PAI software portfolio. It was downloaded onto a Microsoft Excel spreadsheet where means and standard deviations were calculated for the scales and subscales.

\subsection{Instrumentation}

The PAI (Morey, 1991) is self-administered questionnaire that requires roughly 1 hour to complete; the respondent is asked to check one of four response options indicating the extent to which the item statement accurately describes them. Responses range from very true to not at all true. The PAI contains 344 items forming 22 independent scales. It has 4 validity scales, 11 clinical scales (with 3 - 4 subscales each), 5 treatments scales and 2 interpersonal scales. Ten of the clinical scales contain subscales designed to facilitate the full breadth of each complex clinical construct. It assesses validity, clinical, interpersonal style, treatment complications, and subject environment. The PAI is scored using linear, non-transformed t-scores. Mean t-scores are generally 50 with a standard deviation of 10 . Scores of 70+ are generally considered clinically significant, thus these profiles were also examined to include the number reaching clinical significance. A score of greater than or equal to 70 represents a level that is rarely experienced in the general population (Morey \& Quigley, 2002).

The PAI produces a number of scales that are used to help determine the validity of the profile. Two of the main scales used for this purpose are the Positive Impression Management scale and the Negative Impression Management scale. Individuals encounter several forces that can shape their test taking attitudes and sway them to potentially overreport or underreport their symptoms. This response distortion can have a significant impact on the entire profile, and if it is significant enough, it will invalidate the profile.

The clinical scales found on the PAI generally measure symptoms of common mental health conditions to include anxiety, depression, alcohol and drug related concerns, paranoia, mania, psychotic symptoms, anxiety related disorders such as post-traumatic stress or obsessive-compulsive symptoms, somatic symptoms and the like. Additionally, it measures some personality disorder symptoms to include borderline and antisocial personality disorders. The PAI has other scales that measure suicide potential, violence potential, and openness to psychological treatment. It has been found to be a valuable tool for assessment and treatment planning in a number of settings with clients who present with a wide variety of 
presenting problems.

\section{Results}

\subsection{Facilitator Comments}

Themes from the facilitator interviews are presented separately in the paragraphs that follow. Several of the themes were identified and are discussed to illustrate how the facilitators perceived the value of the PAI in the intake process.

Extremely useful. The majority of the facilitators felt that this information was very helpful for them in a number of ways. Facilitators discussed how it helped them to better understand why a group member might be acting a particular way during group. "I was getting frustrated with one group member because they seemed so disengaged from group. I was going to confront him and was considering kicking him out, but thought I'd look at his PAI information first. He showed high on depression, so I checked in with him after group and asked him about it and if he was getting any help for it. He said 'no' so I suggested someone he could talk to and to talk with his doctor about whether he would benefit from an antidepressant. Just the act of doing that ended up making him more connected to me and the group." Another facilitator indicated they found these profiles helpful as well. "I find these profiles extremely useful because it helps me understand some of the issues and challenges that will likely influence my group members ability or desire to change..."

Even invalid profiles were seen as useful for facilitators. One facilitator indicated that he was in a situation where a group member was very defensive during group and appeared to be making little or no progress. The facilitator discussed a situation where he needed to report back to the judge about the group member's progress. "Before I went to see the judge, I was able to look at this group member's PAI information and see that his Positive Impression Management scale score was so high that his profile was invalid. I realized that this was very consistent with how this guy was behaving in group and 12 weeks into group, things hadn't changed. I was able to explain to the judge that we used a psychological test with him when he first started group that indicated very high levels of positive impression management and that this seemed to fit how he was acting in group. It gave me more confidence that it wasn't just a bias of mine, because this meant that the group member ended up going to jail."

Interesting, but the group structure doesn't allow me to use the information. Another theme among the facilitators was that they found the information helpful but they felt so encumbered by the structure of the program they were facilitating that they didn't think they could use this information as well as they wanted to. In other words, they didn't feel like they could customize their intervention to their individual client since they were using a structured group intervention protocol. As an example, one facilitator mentioned "these are interesting, but the group structure makes it hard to do anything other than the standard programming..." Another facilitator indicated that "the group members 
have a number of problems they have to deal with, but we really have to just focus on their IPV. We need to leave the other stuff to someone else to help them manage. I feel like I already have my hands full just trying to help them stop engaging in physical and emotional violence."

Not interested/Disengaged. One facilitator indicated that they were not interested in the PAI information. They frankly outlined the challenges a number of domestic violence programs face when it comes to hiring staff for these challenging positions: “I didn't really look at these at all. I just don't have the time. I don't mean to sound selfish, but I get paid $\$ 14$ an hour to facilitate these groups and I'm a very part-time employee. I already have to work more hours than I get paid for this position and I'm really busy. I'm trying to do the best I can and want to do a good job, but I just don't have the time to go above and beyond what I'm being paid to do..."

Summary. Overall, facilitators appeared to really value the PAI. If they actually took the time to look at the scoring and interpretation, none of the facilitators indicated that they felt like it was a waste of time. Rather, their concerns appeared to be on the fact that the system they were in wasn't flexible enough for them to be able to be more adaptable. While they appeared to generally like the structure of the system they worked out of, they could see that one size didn't fit all and an instrument such as the PAI illustrated important individual factors.

\subsection{PAI Profile Findings}

Profiles were initially tested for validity and 27 profiles (17.5\% of the sample) were found to be invalid and were excluded from further analysis. While this number is rather high, it falls in a relatively normal range, between what has been found in a VA inpatient psychiatric hospital (37\%; Braxton, Calhoun, Williams, \& Boggs, 2007) or service members receiving neuropsychological evaluations for (11\%; Kennedy, Cooper, Reid, Tate, \& Lange, 2015).

Table 1 describes a comparison of the PAI norms found in the sample of domestic violence offenders with norms from the only other PAI norms that have been published for IPV offenders found in the literature at the present (Meade, 2006; Chambers \& Wilson, 2007). These norms were combined into a larger dataset of 246 and recalculated for this study. Additionally, norms from a community sample were also included for comparison (Morey et al., 2011).

Inferential statistics were not used on Table 1 for a number of reasons and it is not clear they would have been particularly meaningful. Descriptive statistics tell a valuable story. For this article, the authors decided that a difference of 5 points was deemed worthy of comment.

When comparing mean $\mathrm{T}$-scores of study participants to published norms for IPV offenders, there are only a small number of differences in the scales listed on Table 1. Study participants scored higher on the Alcohol Problems scale, the Antisocial Behaviors subscale, the Negative Relationships subscale, and the Grandiosity scale. There were also a significantly higher number of invalid profiles in the sample, many due to high levels of positive impression management. 
Table 1. Comparison of PAI norms.

\begin{tabular}{|c|c|c|c|}
\hline \multirow[b]{2}{*}{ PAI Scale or Subscale } & \multicolumn{3}{|c|}{ PAI Norms for IPV Offenders versus Community Sample } \\
\hline & $\begin{array}{c}\text { T-Score means and } \\
\text { standard deviation for Study } \\
\text { Participants }(\mathrm{n}=154)\end{array}$ & $\begin{array}{l}\text { Two study IPV } \\
\text { Offender Norms } \\
\quad(\mathrm{n}=246)\end{array}$ & $\begin{array}{l}\text { Community } \\
\text { Sample }^{* \star}\end{array}$ \\
\hline Inconsistency & $52.33(10.31)$ & $51.92(8.61)$ & $53.78(11.21)$ \\
\hline Infrequency & $55.48(9.55)$ & $53.01(9.26)$ & $53.92(13.16)$ \\
\hline Pos Impression Management & $53.83(10.25)$ & $50.14(8.83)$ & $49.09(9.63)$ \\
\hline Neg Impression Management & $49.21(7.96)$ & $52.14(9.85)$ & $53.44(15.04)$ \\
\hline Traumatic Stress & $51.83(11.01)$ & $49.94(6.57)$ & $50.69(9.84)$ \\
\hline Grandiosity & $54.69(10.25)$ & $49.38(7.21)$ & $53.85(9.86)$ \\
\hline Borderline Features & $52.15(9.28)$ & $49.19(7.29)$ & $53.27(10.05)$ \\
\hline$\wedge$ Affective Instability & $49.61(9.49)$ & $50.77(6.83)$ & $52.98(9.50)$ \\
\hline$\wedge$ Negative Relationships & $55.50(9.84)$ & $49.85(10.11)$ & $51.51(9.90)$ \\
\hline Antisocial Features & $54.89(9.26)$ & $52.46(8.95)$ & $56.59(12.26)$ \\
\hline$\wedge$ Antisocial Behaviors & $60.27(9.68)$ & $49.38(7.83)$ & $54.53(11.97)$ \\
\hline Alcohol Problems & $57.73(15.13)$ & $52.63(6.12)$ & $52.32(11.18)$ \\
\hline Drug Problems & $55.71(13.43)$ & $55.1(9.84)$ & $53.38(12.17)$ \\
\hline Aggression & $50.90(11.15)$ & $56.14(11.64)$ & $52.61(10.54)$ \\
\hline ^Aggressive Attitude & $51.72(10.96)$ & $53.58(9.39)$ & $51.30(9.65)$ \\
\hline$\wedge$ Verbal Aggression & $47.32(9.73)$ & $52.17(9.38)$ & $52.01(10.06)$ \\
\hline ^Physical Aggression & $53.70(10.94)$ & $50.42(7.62)$ & $53.36(11.57)$ \\
\hline Stress & $55.41(10.51)$ & $55.05(7.89)$ & $52.19(10.56)$ \\
\hline Non-Support & $48.82(10.61)$ & $51.34(10.67)$ & $52.50(12.06)$ \\
\hline Treatment Rejection & $49.33(9.14)$ & $49.85(9.51)$ & $49.98(9.26)$ \\
\hline Dominance & $51.91(8.54)$ & $49.87(10.74)$ & $50.40(10.86)$ \\
\hline
\end{tabular}

$\wedge$ Subscale of the main scale above it; ${ }^{*}\left(\right.$ Derived from Meade, 2006; Chambers \& Wilson, 2007); ${ }^{* \star}($ Derived from Morey et al., 2011).

The IPV offender norms were higher on the Aggression scale. When comparing mean T-scores of study participants to the Community Sample, the study participants scored higher on the Alcohol Problems scale and Antisocial Behaviors subscale.

The higher positive impression management scores are consistent with findings suggesting that impression management techniques are often found among DV perpetrators (e.g., Helfritz et al., 2006). Additionally, higher negative relationship subscale scores also seem to make sense, considering that IPV perpetrators may to some extent be unaware that their behavior is outside the norm, and justify their abusive behavior on their perceptions such as negative relationship patterns with their partner (Neighbors, Walker, Mbilinyi, O’Rourke, Edleson, Zegree, \& Roffman. 2010). 
Of note, five clinical scales had large percentages that had a high number of T-scores with clinically significant elevations ( $\geq 70)$ among the study participants and are worth noting. These included the Traumatic Stress (20.2\%), the Antisocial Behaviors (19.7\%), the Stress scale (21\%), the Alcohol Problems scale (17.3\%) and the Drug problems (15.7\%) problems. The findings with alcohol and drug problems appear to be consistent with what was reported on intake from the participants, where $34 \%$ indicated involvement with alcohol or drugs. Additionally, the fact that there was a high number of participants who endorsed a significant level of traumatic stress is consistent with much of the research that suggests a link between traumatic experiences and violent or offending behavior (e.g., Ardino, 2012).

Summary. PAI norms for the study participants looked similar to those published for IPV offenders and even for a community sample. Those scales that appear to be elevated seem to tell an understandable story of a group of individuals who are struggling with a larger amount of substance abuse issues, negative relationships and antisocial behaviors than their counterparts in the community. And when they are court-ordered to treatment and are asked to complete a psychological test as part of their intake assessment, they are understandably wary of being honest and have a higher number of invalid profiles due to high levels of positive impression management as well. This group also appears to be suffering from significant levels of traumatic stress and stress in general, complicating their clinical picture and making it more difficult for them to successfully manage their behavior and learn more adaptive interpersonal skills.

\section{Discussion and Challenges for Future Research and Practice}

This study demonstrates some utility of the PAI for this population. While group comparisons of means are interesting, it appears the real utility of the PAI comes from the individual profiles themselves. For instance, while the mean score for the Traumatic Stress scale in this sample was relatively low, over 20\% of the sample had clinically significant $t$-scores $\geq 70$. This suggests over $1 / 5$ of the sample suffered from several PTSD symptoms and may have benefitted from treatment of this potentially co-morbid condition.

There were also a high number of offenders who endorsed significant levels of antisocial behavior and the clinical literature has been quite pessimistic about treatment for these individuals (Harris \& Rice, 2006). While a high T-score on the antisocial features scale is, in and of itself, insufficient evidence to eliminate an offender from consideration for treatment, it can be a useful part of a comprehensive assessment in order to make those determinations.

Even when profiles are invalid due to defensiveness (such as very high positive impression management scale scores), interviews with IPV group facilitators suggested utility with these invalid profiles. Facilitators valued greater awareness of defensiveness and could adapt their approach to decrease defensiveness. 
Having PAI profile information can also better inform facilitators about specific client needs. Even if they cannot significantly alter the treatment protocol, areas of concern can receive greater emphasis. However, it should be noted that the nature of treatment programs for IPV offenders often limits a facilitator's ability to customize the treatment provided, so these profiles are far less valuable than they would be when providing services in a more individualized context.

Limitations to this research include the small number of facilitators interviewed. Two facilitators terminated employment before the authors were able to conduct interviews with them, so additional facilitator perspectives would be valuable. Future research might consider using the Personality Assessment Screener (see Kelley, Edens, \& Douglas, 2018), a shorter version of the PAI, or potentially selecting out the scales of interest within the PAI rather than requiring IPV offenders complete the entire 344 item PAI.

\section{Conclusion}

There can be significant challenges in the delivery of services to perpetrators of IPV. Several standardized programs have been developed for this population and each year, thousands of individuals volunteer or are court mandated to complete these programs. Yet it is clear that every individual circumstance is unique and the better a facilitator of services understands the distinctive challenges their clientele face, the greater the likelihood they can adapt their services in a way that meets their individual needs. Using measurement tools such as the PAI is one such way to understand the idiosyncratic nature of each client and not fall into the trap of thinking that generalized programs designed to fit everyone will address each participant's needs adequately without some level of customization.

\section{Conflicts of Interest}

The authors declare no conflicts of interest regarding the publication of this paper.

\section{References}

Ardino, V. (2012). Offending Behaviour: The Role of Trauma and PTSD. European Journal of Psychotraumatology, 3, Article No. 18968. https://doi.org/10.3402/ejpt.v3i0.18968

Babcock, J. C., Green, C. E., \& Robie, C. (2004). Does Batterers' Treatment Work? A Meta-Analytic Review of Domestic Violence Treatment. Clinical Psychology Review, 23, 1023-1053. https://doi.org/10.1016/i.cpr.2002.07.001

Bohall, G., Bautista, M. J., \& Musson, S. (2016). Intimate Partner Violence and the Duluth Model: An Examination of the Model and Recommendations for Future Research and Practice. Journal of Family Violence, 31, 1029-1033.

https://doi.org/10.1007/s10896-016-9888-x

Braxton, L. E., Calhoun, P. S., Williams, J. E., \& Boggs, C. D. (2007). Validity Rates of the Personality Assessment Inventory and the Minnesota Multiphasic Personality Inventory-2 in a VA Medical Center Setting, Journal of Personality Assessment, 88, 5-15. https://doi.org/10.1080/00223890709336829 
Census Bureau (2021). American Community Survey (ACS). https://www.census.gov/programs-surveys/acs

Chambers, A. L., \& Wilson, M. N. (2007). Assessing Male Batterers with the Personality Assessment Inventory. Journal of Personality Assessment, 88, 57-65. https://doi.org/10.1080/00223890709336835

Charles, N. E., Floyd, P. N., Bulla, B. A., Barry, C. T., \& Anestis, J. C. (2021). PAI-A Predictors of Treatment Response in a DBT-A-Informed Intervention for Adolescent Boys. Journal of Psychopathology and Behavioral Assessment, 1-14. https://doi.org/10.1007/s10862-021-09886-Z

Cheng, S. Y., Davis, M., Jonson-Reid, M., \& Yaeger, L. (2019). Compared to What? A Meta-Analysis of Batterer Intervention Studies Using Nontreated Controls or Comparisons. Trauma, Violence, \& Abuse, 20, 496-511. https://doi.org/10.1177\%2F1524838019865927

Corvo, K., Dutton, D., \& Chen, W. (2009). Do Duluth Model Interventions with Perpetrators of Domestic Violence Violate Mental Health Professional Ethics? Ethics \& Behavior, 19, 323-340. https://doi.org/10.1080/10508420903035323

Cunliffe, T. B. (2019). A PCL-R, Rorschach, and PAI Investigation of Females with Sex Offenses against Minors and a Rorschach Comparison with Male Pedophiles. Archives of Assessment Psychology, 9, 113-137.

Delsol, C., Margolin, G., \& John, R. S. (2003). A Typology of Maritally Violent Men and Correlates of Violence in a Community Sample. Journal of Marriage and Family, 65, 635-651. https://doi.org/10.1111/j.1741-3737.2003.00635.x

Douglas, H., \& Fitzgerald, R. (2018). The Domestic Violence Protection Order System as Entry to the Criminal Justice System for Aboriginal and Torres Strait Islander people. International Journal for Crime, Justice and Social Democracy, 7, 41-57. https://doi.org/10.5204/ijcjsd.v7i3.499

Edens, J. F., Cruise, K. R., \& Buffington-Vollum, J. K. (2001). Forensic and Correctional Applications of the Personality Assessment Inventory. Behavioral Sciences \& the Law, 19, 519-543. https://doi.org/10.1002/bsl.457

Gardner, B. O., Boccaccini, M. T., Bitting, B. S., \& Edens, J. F. (2015). Personality Assessment Inventory Scores as Predictors of Misconduct, Recidivism, and Violence: A Meta-analytic Review. Psychological Assessment, 27, 534-544.

https://doi.apa.org/doi/10.1037/pas0000065

Harris, G. T., \& Rice, M. E. (2006). Treatment of Psychopathy. In C. J. Patrick (Ed.), Handbook of Psychopathy (pp. 555-572). The Guilford Press.

https://www.gwern.net/docs/algernon/2006-harris.pdf

Helfritz, L. E., Stanford, M. S., Conklin, S. M., \& Greve, K. W. (2006). Usefulness of Self-Report Instruments in Assessing Men Accused of Domestic Violence. The Psychological Record, 56, 171-180. https://doi.org/10.1007/BF03395542

Hsieh, H. F., \& Shannon, S. E. (2005). Three Approaches to Qualitative Content Analysis. Qualitative Health Research, 15, 1277-1288. https://doi.org/10.1177\%2F1049732305276687

Humenik, A. M., Sherrill, B. N., Kantor, R. M., \& Dolan, S. L. (2019). Using the PAI-A to Classify Juvenile Offenders by Adjudicated Offenses. Journal of Child \& Adolescent Trauma, 12, 469-477. https://doi.org/10.1007/s40653-019-00265-1

Jeremiah, R. D., \& Oyewuwo-Gassikia, O. B. (2019). The Public Health Disparities of Domestic Violence: Including the Perspectives and Health of Ethnic-Minority Men. In D. M. Griffith, M. A. Bruce, \& R. J. Thorpe (Eds.), Men's Health Equity (pp. 189-202). New York, NY: Routledge. https://doi.org/10.4324/9781315167428-12 
Kelley, S. E., Edens, J. F., \& Douglas, K. S. (2018). Concurrent Validity of the Personality Assessment Screener in a Large Sample of Offenders. Law and Human Behavior, 42, 156-166. https://doi.apa.org/doi/10.1037/lhb0000276

Kennedy, J. E., Cooper, D. B., Reid, M. W., Tate, D. F., \& Lange, R. T. (2015). Profile Analyses of the Personality Assessment Inventory Following Military-Related Traumatic Brain Injury. Archives of Clinical Neuropsychology, 30, 236-247.

https://doi.org/10.1093/arclin/acv014

Magyar, M. S., Edens, J. F., Lilienfeld, S. O., Douglas, K. S., Poythress Jr., N. G., \& Skeem, J. L. (2012). Using the Personality Assessment Inventory to Predict Male Offenders' Conduct During and Progression through Substance Abuse Treatment. Psychological Assessment, 24, 216-225. https://doi.apa.org/doi/10.1037/a0025359

Meade, K. A. (2006). The Personality Assessment Inventory's Utility in Predicting Domestic Violence Treatment Outcome. Ph.D. Thesis, Minneapolis, MN: Capella University.

https://www.proquest.com/openview/ad8fb00d8594a3b84606c818b3e2dbb2/1?pq-origs $\underline{\text { ite }}=$ gscholar $\& \mathrm{cbl}=18750 \&$ diss $=\mathrm{y}$

Miller, H. A., \& Marshall, E. A. (2019). Comparing Solo-and Co-Offending female Sex Offenders on Variables of Pathology, Offense Characteristics, and Recidivism. Sexual Abuse, 31, 972-990. https://doi.org/10.1177\%2F1079063218791179

Morey, L. C., \& Quigley, B. D. (2002). The Use of the Personality Assessment Inventory (PAI) in Assessing Offenders. International Journal of Offender Therapy and Comparative Criminology, 46, 333-349. https://doi.org/10.1177\%2F0306624X02463007

Morey, L. C., Lowmaster, S. E., Coldren, R. L., Kelly, M. P., Parish, R. V., \& Russell, M. L. (2011). Personality Assessment Inventory Profiles of Deployed Combat Troops: An Empirical Investigation of Normative Performance. Psychological Assessment, 23, 456-462. https://doi.apa.org/doi/10.1037/a0022173

Morey, L.C. (1991). The Personality Assessment Inventory Professional Manual. Lutz, FL: Psychological Assessment Resources. https://doi.apa.org/doi/10.1037/t03903-000

Neighbors, C., Walker, D. D., Mbilinyi, L. F., O’Rourke, A., Edleson, J. L., Zegree, J., \& Roffman, R. A. (2010). Normative Misperceptions of Abuse among Perpetrators of Intimate Partner Violence. Violence against Women, 16, 370-386.

https://doi.org/10.1177\%2F1077801210363608

Pappas, A. P. (2021). Evaluating Antecedents to Treatment Success in Juveniles with Sexually Problematic Behavior. PCOM Psychology Dissertations, No. 540. Philadelphia, PA: Philadelphia College of Osteopathic Medicine.

https://digitalcommons.pcom.edu/psychology dissertations/540

Parker, A. J., Mulay, A. L., \& Gottfried, E. D. (2020). The Personality Assessment Inventory (PAI): Treatment Scales and Interpersonal Characteristics in a Sample of Men Charged with or Convicted of a Sexual Offense. Journal of Forensic Sciences, 65, 2050-2057. https://doi.org/10.1111/1556-4029.14512

Patton, M. Q. (2002). Two Decades of Developments in Qualitative Inquiry: A Personal Experiential Perspective. Qualitative Social Work, 1, 261-283. https://doi.org/10.1177\%2F1473325002001003636

Pence, E., \& Paymar, M. (1993). Education Groups for Men Who Batter: The Duluth Model. New York, NY: Springer Publishing Company, Inc.

https://doi.org/10.1891/9780826179913 